\title{
James, David \& Zöller, Günter (eds.) The Cambridge Companion to Fichte
}

\section{Emiliano Acosta}

\section{(2) OpenEdition \\ Journals}

Edición electrónica

URL: http://journals.openedition.org/ref/721

DOI: $10.4000 /$ ref.721

ISSN: 2258-014X

Editor

EuroPhilosophie Editions

Referencia electrónica

Emiliano Acosta, « James, David \& Zöller, Günter (eds.) The Cambridge Companion to Fichte », Revista de Estud(i)os sobre Fichte [En línea], 13 | 2017, Publicado el 01 marzo 2017, consultado el 25 septiembre 2020. URL : http://journals.openedition.org/ref/721 ; DOI : https://doi.org/10.4000/ref.721

Este documento fue generado automáticamente el 25 septiembre 2020

(c) EuroPhilosophie 


\title{
James, David \& Zöller, Günter (eds.) The Cambridge Companion to Fichte
}

\author{
Emiliano Acosta
}

\section{REFERENCIA}

James, D. \& Zöller, G. (eds.) The Cambridge Companion to Fichte, Cambridge: Cambridge University Press, 2016, 419 pp., ISBN 9780521472265

1 “Fichte tiene finalmente su Companion". Tal vez valga la pena comenzar la presente reseña con estas palabras de Jimena Solé durante la breve presentación de este libro a cargo de Günter Zöller, uno de sus editores, en el IV Congreso Internacional de la Asociación Latinoamericana de Estudios sobre Fichte en Buenos Aires, realizado en septiembre de 2016 (una crónica sobre este evento a cargo de Mariano Gaudio se incluye en este número de la Revista de Estud(i)os sobre Fichte). En esta frase creo leer cierta idea y valoración que muchos miembros de la comunidad académica internacional tienen acerca de esta serie de volúmenes de la Cambridge University Press. En efecto, suele verse en la inclusión de un filósofo en el repertorio de los Cambridge Companions un tipo de reconocimiento al filósofo en cuestión. La doctrina de la ciencia, al igual que la filosofía kantiana, Sócrates o Marx, merecía pues ocupar un lugar en este panteón editorial de la filosofía. En este sentido, la aparición del Companion to Fichte es sin duda un hecho editorial que debe ser celebrado por la comunidad internacional dedicada a la investigación, interpretación y discusión del legado fichteano.

2 La edición ha estado desde el inicio a cargo de Günter Zöller, uno de los más importantes e influyentes comentadores de la doctrina de la ciencia. A este trabajo se sumó en la fase final David James, autor de tres libros, publicados en la misma editorial que el Companion, sobre temas de filosofía política y de derecho en Fichte. Si bien es cierto que la participación de James se restringe a la revisión de las traducciones y de las contribuciones en general -la introducción es de Zöller y James ingresó al proyecto sólo cuando casi todos los autores ya habían sido invitados y la mayoría de los artículos 
ya estaban escritos-, debe mencionarse que el hecho de que el Companion to Fichte haya visto la luz es en gran parte mérito de James quien, según sus palabras, tuvo que reactivar este proyecto que se hallaba años detenido.

Ahora bien, más allá de la verdad que encierran las palabras de Solé, también es cierto que según la editorial de la Universidad de Cambridge el Companion to Fichte no es en primer lugar un premio o reconocimiento, sino antes bien una obra que busca, por un lado, introducir al lector anglosajón que aún no ha leído a Fichte en el pensamiento de este filósofo alemán, y, por otro, ofrecer al especialista en Fichte un panorama de los más recientes desenvolvimientos de la investigación fichteana. En lo que respecta al primer punto, las contribuciones reunidas en el Companion pretenden remediar las dificultades, con las que se enfrenta el lector aún no iniciado en este autor, tan pronto como intenta dar sus primeros pasos en el universo fichteano. En lo que respecta al segundo punto, el Companion quiere servir también como instrumento para la investigación especializada en Fichte. Esta doble finalidad, condicionada por la intención de llegar a dos tipos muy diferentes de público, resulta en un doble discurso o, para decirlo de otro modo, en la existencia de al menos dos registros distintos en los capítulos del Companion to Fichte. Esta dualidad en los niveles de análisis y exposición tiene ciertamente sus ventajas, dado que vuelve al Companion atractivo para distintos grupos de lectores, sin embargo, según mi parecer, trae consigo algunos problemas en lo que concierne a la unidad sistemática y al carácter exhaustivo de este libro. Sobre esta cuestión, que, creo, es un problema inherente a toda obra de este estilo, volveré al final de mi reseña, luego de haber expuesto de manera muy sucinta el contenido mismo del Companion.

Tal como Zöller anuncia en su Introducción, los catorce capítulos del Companion se agrupan básicamente en tres ejes temáticos: surgimiento, desarrollo y recepción de la filosofía de Fichte. El volumen se completa con una introducción, una cronología básica de la vida y obra de Fichte, una breve biografía de los autores, una selección de la bibliografía existente de y sobre Fichte y un índice de nombres y temático.

El primer grupo de capítulos está dedicado al surgimiento de la doctrina de la ciencia. Se trata de dos artículos en los que se analiza el tránsito de Kant a Fichte (Wayne M. Martin) y la relación tanto histórica como conceptual entre la doctrina de la ciencia y la Revolución Francesa (Frederick Beiser). Se trata, sin duda, de dos temas más que conocidos para el especialista en Fichte, sin embargo, debe tenerse aquí en cuenta que ambos capítulos poseen un carácter principalmente introductorio y, que, en este sentido, están destinados a nuevos lectores de Fichte. Martin reconstruye histórica y sistemáticamente el tránsito desde la filosofía kantiana a la concepción fichteana del Yo absoluto a través de un análisis de las contribuciones de Reinhold, Schulze, Creuzer y Fichte al debate acerca de la consistencia y capacidad explicativa de la filosofía transcendental kantiana. Beiser, por su parte, en su artículo sobre Fichte y la Revolución Francesa propone reexaminar la conexión entre los escritos políticos fichteanos de 1793 y el Fundamento de toda la doctrina de la ciencia de 1794/95 postulando como elemento mediador la recepción positiva (¿optimista, tal vez?) que en esos años hace Fichte de la Revolución Francesa. Si bien este artículo es de carácter introductorio, Beiser ofrece aquí argumentos convincentes para sostener la tesis de que el último fin de la doctrina de la ciencia es político, es decir, que la comparación que hace Fichte entre su sistema de filosofía y la Revolución Francesa es algo más que un gesto literario. La crítica fichteana de la cosa en sí no es mera filosofía especulativa, según Beiser, sino 
la base para la deconstrucción de la ideología del sistema político y religioso reinante en la Alemania de la época.

6 El segundo grupo lo componen por un lado los capítulos dedicados al desarrollo histórico de la doctrina de la ciencia en sentido restringido, es decir como filosofía primera, y de las disciplinas filosóficas que componen el sistema fichteano en sentido amplio. En el primer subgrupo se examina la doctrina de la ciencia en sus distintas presentaciones de 1794 a 1814. En el segundo, cuestiones concernientes a la filosofía del derecho y de las costumbres, a la economía política, a la filosofía de la historia, a la cuestión del nacionalismo y a la filosofía de la religión.

7 Las distintas versiones del sistema fichteano constituyen el tema de las contribuciones de Christian Klotz sobre el Fundamento de la doctrina de la ciencia, de Daniel Breazeale sobre la doctrina de la ciencia nova método y de Günter Zöller sobre la doctrina de la ciencia entre 1800 y 1814. Estos tres artículos buscan brindar una explicación del desarrollo todo de la doctrina de la ciencia en sus distintos momentos. Como puede verse, no se han tenido en cuenta las divisiones habituales de la doctrina de la ciencia en un antes y después de Jena o en un primer periodo: los escritos de Jena entre 1794 y 1799, un segundo periodo entre Berlín y Erlangen de 1800 a 1805 o 1806, y un tercer período incluyendo o comenzando con la doctrina de la ciencia de Königsberg y culminando en las últimas lecciones de Fichte en Berlín en 1814. En su artículo "Sistema y Vida: El legado filosófico de Fichte" Zöller ha explicado por qué estos esquemas interpretativos ya no pueden ser más defendidos. Sencillamente se trata de una cuestión de información: la evidencia de la que disponemos gracias a la publicación de la totalidad de los manuscritos de Fichte no nos permite dividir la obra de Fichte en los términos en que se hacía hasta hace unos veinte años. Los editores han optado por analizar el desarrollo de la doctrina de la ciencia tomando como puntos principales la primera y única versión impresa durante la vida de Fichte de su sistema, la exposición de su sistema entre 1796 y 1799 y lo propiamente nuevo de las versiones posteriores a Jena, a saber: la recepción y apropiación fichteana de las críticas de Jacobi y Schelling y el carácter metafilosófico de la doctrina de la ciencia en sus últimas versiones. En estos tres artículos el lector encontrará una visión de conjunto de las distintas versiones de un mismo proyecto, las cuales difieren, sin duda, en lo que se refiere al método de exposición y al foco de análisis, pero en modo alguno dan testimonio de un cambio dramático o sustancial en la obra filosófica de Fichte.

8 Este subgrupo de artículos es seguido por el análisis y la discusión de los escritos fichteanos sobre disciplinas filosóficas o temáticas específicas. Allen W. Wood ofrece un detallado estudio de la filosofía del derecho y de la ética fichteanas. Si bien su exposición de las ideas principales de Fichte en lo que concierne a estos ámbitos del saber es más que satisfactoria, la explicación que Wood da de cuestiones tan sensibles y centrales como la teoría del reconocimiento y el derecho de rebelión no resulta, según mi parecer, del todo satisfactoria. En ambos casos se trata del mismo problema: un análisis a partir de un corpus que no es exhaustivo. De allí que Wood no problematice las dos deducciones del reconocimiento que Fichte da en esta obra ni vea en la teoría fichteana del derecho a la rebelión la verdadera intención de Fichte, la cual, cuando es leída a la luz de la respuesta de Fichte a una reseña anónima de su Fundamento del derecho natural, no es, como Wood señala, tornar la rebelión imposible, sino antes bien darle a la misma un marco constitucional. Jean-Christophe Merle es el autor del capítulo dedicado a la economía política. Se trata de una discusión del Estado comercial 
cerrado en combinación con El fundamento del derecho natural en la que Merle explica por qué el proyecto fichteano de economía política en modo alguno es una utopía y ofrece un interesante análisis del concepto fichteano de propiedad. A las exposiciones de temas específicos de la filosofía de Fichte se suma el aporte de Ives Radrizzani sobre el compromiso histórico de la doctrina de la ciencia. Radrizzani muestra la tensión interna en el pensamiento de Fichte entre el nivel a priori de la doctrina de la ciencia en sentido estricto y la dimensión inherente o necesaria de la historicidad que esta filosofía posee en cuanto instrumento de ennoblecimiento moral de la vida humana en la tierra. Si bien el artículo siguiente, a cargo de Alexander Aichele, vuelve a tratar el tema de la historicidad y de la filosofía de la historia, su tema específico es la cuestión del nacionalismo en los Discursos a la nación alemana de Fichte. En el texto de Aichele se destaca el análisis que ofrece del escrito de Fichte sobre Maquiavelo y la relación que establece entre este escrito, Los caracteres de la edad contemporánea y los Discursos. Considero, sin embargo, un elemento innecesario en este trabajo su apología del chauvinismo fichteano, consistente en afirmar que no se trata de racismo, pues Fichte no introduce elementos étnicos o biológicos en su argumentación sobre la supremacía de la alemanidad. Aichele repite un argumento que sólo puede ser tolerado en algunos círculos fichteanos. Este subgrupo se cierra con el capítulo acerca de la filosofía de la religión de Fichte a cargo de Hansjürgen Verweyen. Verweyen muestra cómo la religión o el motivo de lo religioso en la constitución de la individualidad racional no sólo atraviesa toda la obra de Fichte, sino que además representa el momento último en la búsqueda del conocimiento y experiencia de lo absoluto en la finitud humana. Si bien la lectura de Verweyen de la teoría fichteana de los cinco modos de considerar el mundo en su Instrucciones para la vida bienaventurada puede ser discutida y la comparación entre Fichte y Anselmo de Canterbury tiene sus raíces en realidad en la formación de Verweyen y no en la filosofía de Fichte, su reconstrucción histórica y conceptual de la filosofía de la religión de Fichte ofrece una visión de conjunto no sólo de este tema sino de la doctrina de la ciencia en general. Instructivo resulta también en este capítulo el tratamiento que Verweyen hace de la influencia de Lessing en el pensamiento de Fichte.

9 Los artículos del tercer y último grupo aparecen anunciados en la introducción como aportes acerca de la recepción de la filosofía fichteana en el romanticismo alemán (Elizabeth Millán), en Schelling (Sebastian Gardner), en la teoría del reconocimiento hegeliana (David James) y en la así llamada philosophy of mind (Paul Franks). Si bien es cierto que el primero de los artículos se concentra propiamente en la cuestión de la recepción de la doctrina de la ciencia (en Hölderlin y F. Schlegel), en los otros tres la cuestión propia de la recepción es secundaria o inexistente. Gardner analiza los encuentros y desencuentros entre Fichte y Schelling. Entre los puntos principales de su trabajo se hallan la intención de mostrar que el Schelling fichteano de Jena nunca fue un verdadero fichteano y que no es Fichte sino otros filósofos quienes en verdad conforman el pensamiento de Schelling (Platón y Kant). James, por su parte, se dedica a la cuestión acerca del modo en que la esclavitud aparece concebida a partir de las teorías de reconocimiento de Fichte y Hegel. Por último, Franks ofrece principalmente una argumentación tan clara como convincente contra la tesis que sostiene que Fichte es subjetivista, mostrando así que la doctrina de la ciencia no puede ser considerada una filosofía internalista, conflacionista ni creativista. De más está decir que el hecho de que en estos tres artículos no sea tratada la cuestión de la recepción de la filosofía de Fichte en sentido estricto en modo alguno quita valor a estas contribuciones. Sin 
embargo, la selección de los mismos para el Companion to Fichte vuelve visible uno de los principales problemas inherentes a este tipo de volúmenes colectivos, a saber: la imposibilidad de lograr que el libro se vuelva una unidad o que el mismo logre cubrir de modo introductorio todos los aspectos del filósofo en cuestión, en el presente caso Fichte.

10 Si bien es cierto que es indiscutible la necesidad de incluir en un Companion capítulos sobre todas las distintas versiones de la doctrina de la ciencia como también acerca de las disciplinas filosóficas que conforman el plan originario de un sistema de ciencias filosóficas en Fichte; no se da el mismo consenso inmediato en el caso de la decisión de, por un lado, incluir un estudio sobre la recepción de Fichte en Schlegel y Novalis pero, por otro, no dedicar un capítulo a la recepción de Fichte en Reinhold y Bardili. Lo mismo podría decirse de la decisión tanto de no incluir cuestiones centrales para comprender el desarrollo histórico y conceptual de la doctrina de la ciencia tales como la disputa del ateísmo, la pertenencia y actividad masónica de Fichte y la cuestión del antisemitismo, como de restringir la recepción de Fichte en nuestros días al caso de la filosofía de la mente. Con respecto a esto último, por cierto, podría alegarse con todo derecho que el Companion está pensado para el público anglosajón y es el interés de este público el que determina qué pasos a seguir en estas cuestiones. No se trata aquí de criticar la decisión de los editores, sino de mostrar el problema que todo Cambridge Companion (el de Fichte no es una excepción) trae consigo.

11 Dejando de lado la discusión acerca de las razones para la inclusión de artículos que ni versan sobre la doctrina de la ciencia en general ni sobre las aplicaciones particulares elaboradas por Fichte, creo necesario confesar mi sorpresa al notar que no hay un capítulo dedicado a la concepción fichteana de la naturaleza o a una reconstrucción de lo que podría ser llamado filosofía de la naturaleza en Fichte. La filosofía de la naturaleza, siguiendo a W. Janke, conforma junto con la ética, la filosofía del derecho, la filosofía de la religión y la doctrina de la ciencia en sentido estricto la estructura quíntuple básica de la idea de sistema de ciencias filosóficas de Fichte. Sabemos que Fichte nunca escribió un tratado sobre la naturaleza, pero sabemos también que tampoco escribió una filosofía de la religión en el modo en que lo hizo acerca del derecho y de la moral. Creo que el Companion se habría enriquecido aún más con un capítulo que expusiera histórica y sistemáticamente los modos en que Fichte concibe la naturaleza. De que sobre este tema contamos con muy buenos especialistas lo muestra el volumen colectivo 'Natur' in der Transzendentalphilosophie: Eine Tagung zum Gedenken an Reinhard Lauth. (Berlin: Duncker \& Humblot, 2015).

12 Con respecto al trabajo técnico de la edición creo que el lector habría estado agradecido si se hubieran introducido referencias internas al libro, es decir, entre los distintos capítulos, de modo que los autores no se hubieran visto obligados a volver a explicar cuestiones que ya habían sido tratadas en otros capítulos. El índice temático debe ser revisado, pues en algunos casos sólo da cuenta del uso de un término en uno de los capítulos y no en todo el libro. Así, por ejemplo, en los casos de 'cobardía' (cowardice) y 'pereza' (laziness) el índice conduce al lector únicamente al texto de James, cuando en realidad estos conceptos son explicados en detalle en el artículo de Wood sobre derecho y moral. La bibliografía no es exhaustiva, pero ciertamente no era éste el fin de la misma. Creo ver que el criterio ha sido sobre todo el hecho de que el Companion se dirige al público de lengua inglesa. El lector encontrará allí obras de consulta fundamentales, como las de Léon, Gueroult, Janke, Henrich y Zöller. 
13 Sin duda alguna, resulta tarea fácil enumerar lo que este Companion no ofrece, pero una obra se juzga por lo que es y no por lo que uno desearía que fuera. En este sentido debe decirse que el Companion to Fichte ofrece al neófito y al especialista estudios introductorios claros y a cargo de reconocidos especialistas tanto sobre la doctrina de la ciencia en sus distintas versiones, así como también sobre su filosofía del derecho, ética, economía política y concepción de la historia y de la religión. Se trata pues de una herramienta útil de consulta que muestra sus verdaderas ventajas al ser utilizada como complemento del estudio de la obra misma de Fichte.

\section{AUTORES}

\section{EMILIANO ACOSTA}

Ghent University/Vrije Universiteit Brussel 